\title{
A USEFUL ORTHONORMAL BASIS ON BI-SLANT SUBMANIFOLDS OF ALMOST HERMITIAN MANIFOLDS
}

\author{
MEHMET GÜLBAHAR, EROL KILIÇ AND SADIK KELEŞ
}

\begin{abstract}
In this paper, we study bi-slant submanifolds of an almost Hermitian manifold for different cases. We introduce a new orthonormal basis on bi-slant submanifold, semi-slant submanifold and hemi-slant submanifold of an almost Hermitian manifold to compute Chen's main inequalities. We investigate these inequalities for semi-slant submanifolds, hemi-slant submanifolds and slant submanifolds of a generalized complex space form. We obtain some characterizations on such submanifolds of a complex space form.
\end{abstract}

\section{Introduction}

The theory of submanifolds of an almost Hermitian manifold or a Kaehlerian manifold began as a separate field of study in the last century with the investigation of algebraic curves and algebraic surfaces in classical algebraic geometry. In the early 1950s, invariant submanifolds of an almost Hermitian manifold were defined by E. Calabi [2, 3] and in the early 1970s, anti-invariant submanifolds were defined by B. Y. Chen and K. Ogiue [6] as follows:

Let $M$ be a submanifold of an almost Hermitian manifold $(\widetilde{M}, J, \widetilde{g})$. For any $X \in T_{p} M, J X$ can be decomposed into tangential and normal parts given by

$$
J X=T X+F X, P X \in T_{p} M, F X \in T_{p}^{\perp} M,
$$

where $T X$ is the tangential component and $F X$ is the normal component of $J X$. The manifold $M$ is called an invariant submanifold if $F=0$ and anti-invariant submanifold if $T=0$.

In 1990, B.-Y. Chen [7] introduced slant submanifolds as a generalization of invariant submanifold and anti-invariant submanifolds as follows:

For a vector $0 \neq X_{p} \in T_{p} M$, if the angle $\theta\left(X_{p}\right)$ between $J X_{p}$ and $X_{p}$ is independent of the choice of point $p \in M$, then $M$ is called a slant submanifold. Invariant submanifolds and anti-invariant submanifolds are slant submanifolds with $\theta=0$ and $\theta=\frac{\pi}{2}$, respectively.

Received September 23, 2014, accepted October 19, 2015.

2010 Mathematics Subject Classification. 53C15, 53C40.

Key words and phrases. Almost Hermitian manifold, Slant submanifold, Complex space form.

Corresponding author: Mehmet Gülbahar. 
Furthermore, slant distribution was introduced in [1] by J. L. Cabrerizo et al., as follows:

A differentiable distribution $D$ on $M$ is called a slant distribution, if for each $p \in M$ and each non-zero vector $X \in D_{p}$, the angle $\theta_{D}(X)$ between $J X$ and $X$ is constant and is independent of the choice of $p \in M$ and $X \in D_{p}$.

In 2002, the notion of bi-slant submanifolds of an almost Hermitian manifold was introduced as a natural generalization of semi-slant submanifolds by A. Carriazzo [4] as follows:

There exist two orthogonal distributions $D_{1}$ and $D_{2}$ on $M, \operatorname{dim} D_{1}=2 d_{1}$ and $\operatorname{dim} D_{2}=$ $2 d_{2}$ such that

(i) $T M=D_{1} \oplus D_{2}$,

(ii) $D_{1}$ and $D_{2}$ are slant distributions with $\theta_{1}$ and $\theta_{2}$ angles, respectively.

Semi-slant submanifolds, hemi-slant submanifolds, CR-submanifolds, slant submanifolds are particular cases of bi-slant submanifolds. In fact, $M$ is a semi-slant submanifold if $D_{1}$ is an invariant distribution and $D_{2}$ is a slant distribution, $M$ is a hemi-slant submanifold if $D_{1}$ is a slant distribution and $D_{2}$ is an anti-invariant distribution, $M$ is a CR-submanifold if $\theta_{1}=0$ and $\theta_{2}=\frac{\pi}{2}, M$ is a slant submanifold if $D_{1}$ or $D_{2}$ is equal to zero.

In [15] and [16], the authors consider an orthonormal basis $\left\{e_{1}, \ldots, e_{n}\right\}$ of an $n$-dimensional bi-slant submanifold on generalized complex space forms to compute Chen inequalities on complex space forms and Sasakian space forms such that this basis satisfies

$$
\begin{aligned}
e_{1}, e_{2} & =\frac{1}{\cos \theta_{1}} T e_{1}, \ldots, e_{2 m-1}, e_{2 m}=\frac{1}{\cos \theta_{1}} T e_{2 m-1}, \\
e_{2 m+1}, e_{2 m+2} & =\frac{1}{\cos \theta_{2}} T e_{2 m+1}, \ldots, e_{2 n-2 m}=\frac{1}{\cos \theta_{2}} T e_{2 n-2 m-1},
\end{aligned}
$$

where $\operatorname{dimD}_{1}=2 m$ and $d i m D_{2}=2 n-2 m$. Here, $T e_{i}$ is perpendicular to $D_{2}$ and $T e_{j}$ is perpendicular to $D_{1}$ for $i \in\{1, \ldots, 2 m\}$ and $j \in\{2 m+1, \ldots, 2 n\}$. But one can not know the angle between $J D_{1}$ and $D_{2}$ or $J D_{2}$ and $D_{1}$ for bi-slant submanifold of almost Hermitian manifolds. Therefore, this basis isn't true for bi-slant submanifolds. For this reason, we introduce a useful basis on bi-slant submanifolds in this study. Using this basis, we compute Chen inequalities and give some corollaries on bi-slant submanifolds of an almost Hermitian manifold.

The paper has been organized as follows: Section 2 is devoted to preliminaries. In section 3 , we give some examples for different cases on bi-slant submanifolds of an almost Hermitian manifold. We introduce an orthonormal basis for bi-slant submanifolds, semi-slant submanifolds, hemi-slant submanifolds, slant submanifolds. In section 4, we establish a sharp inequality involving the mean curvature vector and the Ricci curvature of bi-slant submanifolds. We investigate this inequality for semi-slant submanifolds, hemi-slant submanifolds 
and slant submanifolds of a generalized complex space form. In section 5, we establish an optimal inequality involving the Chen-invariant for bi-slant submanifolds of a generalized complex space form. We study this inequality for some special submanifolds of a generalized complex space form.

\section{Preliminaries}

Let $(\widetilde{M}, \widetilde{g})$ be a $k$-dimensional Riemannian manifold with a Riemannian metric $\widetilde{g}$ and $(M, g)$ be an $n$-dimensional submanifold of $(\widetilde{M}, \widetilde{g})$ with the induced metric tensor $g$. We denote the inner product of both the metrics by $\langle$,$\rangle . Let \sigma$ be the second fundamental form related to the shape operator $A$ by $\langle\sigma(X, Y), N\rangle=\left\langle A_{N} X, Y\right\rangle$. The Gauss equation is given by

$$
\begin{aligned}
R(X, Y, Z, W)= & \widetilde{R}(X, Y, Z, W)+\langle\sigma(X, W), \sigma(Y, Z)\rangle \\
& -\langle\sigma(X, Z), \sigma(Y, W)\rangle
\end{aligned}
$$

for all $X, Y, Z, W \in \Gamma(T M)$, where $\widetilde{R}$ and $R$ are the Riemann curvature tensors of $\widetilde{M}$ and $M$, respectively.

The mean curvature vector $H$ of the submanifold $M$ is given by $H=\frac{1}{n} \operatorname{trace}(\sigma)$. If $\sigma=0$, then the submanifold is called totally geodesic in $\widetilde{M}$, if $H=0$, then the submanifold is called minimal, if $\sigma(X, Y)=g(X, Y) H$ for all $X, Y \in \Gamma(T M)$, then the submanifold is called totally umbilical [19].

Let $\left\{e_{1}, \ldots, e_{n}\right\}$ be an orthonormal basis of the tangent space $T_{p} M$ and $e_{r}$ belongs to an orthonormal basis $\left\{e_{n+1}, \ldots, e_{m}\right\}$ of the normal space $T_{p}^{\perp} M$. We put

$$
\sigma_{i j}^{r}=\left\langle\sigma\left(e_{i}, e_{j}\right), e_{r}\right\rangle \text { and }\|\sigma\|^{2}=\sum_{i, j=1}^{n}\left\langle\sigma\left(e_{i}, e_{j}\right), \sigma\left(e_{i}, e_{j}\right)\right\rangle .
$$

We denote by $K_{i j}$ and $\widetilde{K}_{i j}$ the sectional curvature of the plane section spanned by $e_{i}$ and $e_{j}$ at point $p$ in the submanifold $M$ and in the ambient manifold $\widetilde{M}$, respectively. In this case, using the Gauss equation, we get

$$
K_{i j}=\widetilde{K}_{i j}+\sum_{r=n+1}^{m}\left(\sigma_{i i}^{r} \sigma_{j j}^{r}-\left(\sigma_{i j}^{r}\right)^{2}\right) .
$$

From (2.3), it follows that

$$
2 \tau(p)=2 \widetilde{\tau}\left(T_{p} M\right)+n^{2}\|H\|^{2}-\|\sigma\|^{2} .
$$

Also, the squared second fundamental form and the squared mean curvature satisfy that

$$
\|\sigma\|^{2}=\frac{1}{2} n^{2}\|H\|^{2}+\frac{1}{2} \sum_{r=n+1}^{m}\left(\sigma_{11}^{r}-\sigma_{22}^{r}-\cdots-\sigma_{n n}^{r}\right)^{2}
$$




$$
+2 \sum_{r=n+1}^{m} \sum_{j=2}^{n}\left(\sigma_{1 j}^{r}\right)^{2}-2 \sum_{r=n+1}^{m} \sum_{2 \leq i<j \leq n}\left(\sigma_{i i}^{r} \sigma_{j j}^{r}-\left(\sigma_{i j}^{r}\right)^{2}\right) .
$$

Let $(\widetilde{M}, J, \widetilde{g})$ be an almost Hermitian manifold and $\widetilde{\nabla}$ be the Riemannian connection of the Riemannian metric $\widetilde{g}$. The manifold is called

1. a nearly Kaehler manifold [9] if

$$
\left(\widetilde{\nabla}_{X} J\right) X=0
$$

for any vector field $X \in T \widetilde{M}$,

2. a Kaehler manifold [19] if

$$
\widetilde{\nabla} J=0 .
$$

An almost Hermitian manifold with the $J$-invariant Riemannian curvature tensor $\widetilde{R}$, that is,

$$
\widetilde{R}(J X, J Y, J Z, J W)=\widetilde{R}(X, Y, Z, W), \quad X, Y, Z, W \in \Gamma(T \widetilde{M}),
$$

is called an $R K$-manifold [18].

An almost Hermitian manifold $\widetilde{M}$ is said to have (pointwise) constant type if for each $p \in$ $\widetilde{M}$ and for all $X, Y, Z \in T_{p} \widetilde{M}$ such that

$$
\begin{gathered}
\langle X, Y\rangle=\langle X, Z\rangle=\langle X, J Y\rangle=\langle X, J Z\rangle=0 \text { and } \\
\langle Y, Y\rangle=1=\langle Z, Z\rangle .
\end{gathered}
$$

And consequently, we have

$$
\widetilde{R}(X, Y, X, Y)-\widetilde{R}(X, Y, J X, J Y)=\widetilde{R}(X, Z, X, Z)-\widetilde{R}(X, Z, J X, J Z) .
$$

It is known that if $\widetilde{M}$ is an $R K$-manifold, then it has (pointwise) constant type if and only if there is a differentiable function $\alpha$ on $\widetilde{M}$ satisfying

$$
\widetilde{R}(X, Y, X, Y)-\widetilde{R}(X, Y, J X, J Y)=\alpha\left(\langle X, X\rangle\langle Y, Y\rangle-\langle X, Y\rangle^{2}-\langle X, J Y\rangle^{2}\right)
$$

for all $X, Y, Z \in T \widetilde{M}$. Furthermore, $\widetilde{M}$ has global constant type if $\alpha$ is constant. The function $\alpha$ is called the constant type of $\widetilde{M}$ [17].

A $R K$-manifold of constant holomorphic sectional curvature $c$ and constant type $\alpha$ is denoted by $\widetilde{M}(c, \alpha)$. The Riemann curvature of $\widetilde{M}(c, \alpha)$ is given by

$$
\begin{aligned}
4 \widetilde{R}(X, Y) Z= & (c+3 \alpha)\{\langle Y, Z\rangle X-\langle X, Z\rangle Y\} \\
& +(c-\alpha)\{\langle X, J Z\rangle J Y-\langle Y, J Z\rangle J X
\end{aligned}
$$




$$
+2\langle X, J Y\rangle J Z\}
$$

for all $X, Y, Z \in T \widetilde{M}$. If $c=\alpha$, then $\widetilde{M}(c, \alpha)$ is a space of constant curvature. If $\alpha=0$, then $\widetilde{M}(c)$ is a complex space form.

\section{Bi-slant submanifolds}

Let $M$ be a $2 n$-dimensional bi-slant submanifold of an almost Hermitian manifold $\widetilde{M}$ such that

$$
T M=D_{1} \oplus D_{2},
$$

where $D_{1}$ is a $\theta_{1}$-slant distribution, and $D_{2}$ is a $\theta_{2}$-slant distribution. Then there exist the following four cases [5]:

Case 1: $M$ is bi-slant with $\theta_{1}=\theta_{2}=\theta$ and it is also $\theta$-slant.

Case 2: $M$ is bi-slant with $\theta_{1}=\theta_{2}$ but it is not slant.

Case 3: $M$ is bi-slant with $\theta_{1}=\theta_{2}=\theta$ and it is also $\alpha$-slant with $\alpha \neq 0$.

Case 4: $M$ is bi-slant with $\theta_{1} \neq \theta_{2}$ and it is not slant.

Now, we are going to give an example of bi-slant submanifold for Case $\mathbf{1}$ as follows:

Example 3.1. Let $J$ be an almost complex structure on $R^{6}$ such that

$$
J\left(x_{1}, x_{2}, x_{3}, x_{4}, x_{5}, x_{6}\right)=\left(x_{2},-x_{1}, x_{4},-x_{3}, x_{6},-x_{5}\right) .
$$

Let $M$ be a submanifold of $R^{6}$ given by

$$
\varphi(u, v, w, t)=(u \sqrt{2}, v \sqrt{2}, u+v, u-v, w-t, t-w) .
$$

Then we have an orthonormal frame of $M$ as follows:

$$
\begin{aligned}
X_{1} & =\frac{1}{2}\left(\sqrt{2} \frac{\partial}{\partial x_{1}}+\frac{\partial}{\partial x_{3}}+\frac{\partial}{\partial x_{4}}\right), & X_{2} & =\frac{1}{2}\left(\sqrt{2} \frac{\partial}{\partial x_{2}}+\frac{\partial}{\partial x_{3}}-\frac{\partial}{\partial x_{4}}\right), \\
X_{3} & =\frac{1}{\sqrt{2}}\left(\frac{\partial}{\partial x_{5}}-\frac{\partial}{\partial x_{6}}\right), & X_{4} & =\frac{1}{\sqrt{2}}\left(-\frac{\partial}{\partial x_{5}}+\frac{\partial}{\partial x_{6}}\right) .
\end{aligned}
$$

Put $D_{1}=\operatorname{Span}\left\{X_{1}, X_{2}\right\}$ and $D_{2}=\operatorname{Span}\left\{X_{3}, X_{4}\right\}$, then $M$ is a bi-slant submanifold with $D_{1}$ and $D_{2}$ are anti-invariant distributions. Furthermore, $M$ is an anti-invariant submanifold.

Now, we are going to give an example of bi-slant submanifold for Case $\mathbf{2}$ as follows: 
Example 3.2. We consider the Euclidian space $R^{6}$ with coordinates $\left(x_{1}, x_{2}, x_{3}, x_{4}, x_{5}, x_{6}\right)$. Let $J$ be an almost complex structure on $R^{6}$ such that

$$
J\left(x_{1}, x_{2}, x_{3}, x_{4}\right)=\left(-x_{4},-x_{5},-x_{6}, x_{1}, x_{2}, x_{3}\right) .
$$

Let $M$ be a submanifold of $R^{6}$ with

$$
\begin{aligned}
\varphi\left(u_{1}, u_{2}, v_{1}, v_{2}\right)= & \left(u_{1} \cos \theta_{1}-u_{2} \sin \theta_{1}, u_{1} \sin \theta_{1}+u_{2} \cos \theta_{1}, 0,\right. \\
& \left.v_{1} \cos \theta_{2}-v_{2} \sin \theta_{2}, v_{1} \sin \theta_{2}+v_{2} \cos \theta_{2}, 0\right),
\end{aligned}
$$

for any $\theta_{1}, \theta_{2} \in\left[0, \frac{\pi}{2}\right]$. Then we have an orthonormal frame of $M$ as follows:

$$
\begin{aligned}
& X_{1}=\cos \theta_{1} \frac{\partial}{\partial x_{1}}+\sin \theta_{1} \frac{\partial}{\partial x_{2}}, \quad X_{2}=\cos \theta_{2} \frac{\partial}{\partial x_{4}}+\sin \theta_{2} \frac{\partial}{\partial x_{5}}, \quad X_{3}=\frac{\partial}{\partial x_{3}}, \\
& X_{4}=\frac{\partial}{\partial x_{6}}, \quad X_{5}=-\sin \theta_{1} \frac{\partial}{\partial x_{1}}+\cos \theta_{1} \frac{\partial}{\partial x_{2}}, \quad X_{6}=-\sin \theta_{2} \frac{\partial}{\partial x_{4}}+\cos \theta_{2} \frac{\partial}{\partial x_{5}} .
\end{aligned}
$$

If we put $D_{1}=\operatorname{Span}\left\{X_{1}, X_{2}\right\}, D_{2}=\operatorname{Span}\left\{X_{5}, X_{6}\right\}$, then $M$ is bi-slant submanifold with $D_{1}$ and $D_{2}$ are $\theta=\left(\theta_{1}-\theta_{2}\right)-$ slant distributions. But $M$ is not a slant submanifold.

Now, we are going to give an example of bi-slant submanifold for Case $\mathbf{3}$ as follows:

Example 3.3. Let $J$ be an almost complex structure on $R^{8}$ such that

$$
J\left(x_{1}, x_{2}, x_{3}, x_{4}, x_{5}, x_{6}, x_{7}, x_{8}\right)=\left(-x_{5},-x_{6},-x_{7},-x_{8}, x_{1}, x_{2}, x_{3}, x_{4}\right) .
$$

Let $M$ be a submanifold of $R^{8}$ with

$$
\varphi(u, v, w, t)=\left(\frac{1}{2}(u-v), \frac{1}{2}(u+v), \frac{\sqrt{2}}{2} u, \frac{\sqrt{2}}{2} v, w, t, 0,0\right) .
$$

Then we have an orthonormal frame of $M$ as follows:

$$
\begin{aligned}
& X_{1}=\frac{1}{2}\left(\frac{\partial}{\partial x_{1}}+\frac{\partial}{\partial x_{2}}+\sqrt{2} \frac{\partial}{\partial x_{3}}\right), \quad X_{2}=\frac{1}{2}\left(-\frac{\partial}{\partial x_{1}}+\frac{\partial}{\partial x_{2}}+\sqrt{2} \frac{\partial}{\partial x_{4}}\right), \\
& X_{3}=\frac{\partial}{\partial x_{5}}, X_{4}=\frac{\partial}{\partial x_{6}} .
\end{aligned}
$$

Put $D_{1}=\operatorname{Span}\left\{X_{1}, X_{2}\right\}$ and $D_{2}=\operatorname{Span}\left\{X_{3}, X_{4}\right\}$ then $M$ is bi-slant submanifold with $D_{1}$ and $D_{2}$ are anti-invariant distributions. Futhermore, $M$ is a slant submanifold with slant angle $\theta=\frac{\pi}{3}$.

Let $P_{i}: T M \rightarrow D_{i}, i \in\{1,2\}$, be orthogonal projections. It is well known that

$$
\left\langle P_{i} X_{i}, Y_{i}\right\rangle=\left\langle X_{i}, P_{i} Y_{i}\right\rangle
$$

for $X_{i}, Y_{i} \in \Gamma\left(D_{i}\right)[5,10]$. Furthermore, it can be proved that

$$
\left(P_{i} T\right)^{2} X_{i}=-\cos ^{2} \theta_{i} X_{i}
$$


for any $X_{i} \in \Gamma\left(D_{i}\right)$. From (3.2) and (3.3), we have

$$
\begin{aligned}
\left\langle P_{i} T X_{i}, P_{i} T Y_{i}\right\rangle & =\left\langle T X_{i}, P_{i} T Y_{i}\right\rangle \\
& =-\left\langle X_{i}, T P_{i} T Y_{i}\right\rangle \\
& =-\left\langle X_{i}, P_{1} T P_{i} T Y_{i}+P_{2} T P_{i} T Y_{i}\right\rangle \\
& =-\left\langle X_{i},-\cos ^{2} \theta_{i} Y_{i}\right\rangle \\
& =\cos ^{2} \theta_{i}\left\langle X_{i}, Y_{i}\right\rangle
\end{aligned}
$$

Since $\|T X\|^{2}+\|F X\|^{2}=\|J X\|^{2}$, we get

$$
\left\|P_{1} T X\right\|^{2}+\left\|P_{2} T X\right\|^{2}+\|F X\|^{2}=\|J X\|^{2}
$$

for any $X \in T M$. Now, we choose orthonormal basis $\left\{e_{1}, \ldots, e_{n}\right\}$ of $T_{p} M$ such that $D_{1}=\operatorname{Span}\left\{e_{1}, \ldots, e_{2 m}\right\}$ and $D_{2}=\operatorname{Span}\left\{e_{2 n+1}, \ldots, e_{2 n}\right\}$. Using (3.4) and (3.5), we have

$$
\left\|P_{2} T e_{i}\right\|^{2}+\left\|F e_{i}\right\|^{2}=\sin ^{2} \theta_{1}
$$

and

$$
\left\|P_{1} T e_{j}\right\|^{2}+\left\|F e_{j}\right\|^{2}=\sin ^{2} \theta_{2}
$$

for $i \in\{1, \ldots, 2 m\}$ and $j \in\{2 m+1, \ldots, 2 n\}$. Therefore, we can choose a bi-slant orthonormal basis $\left\{e_{1}, \ldots, e_{2 n}\right\}$ of $T_{p} M$ satisfying that

$$
\begin{aligned}
T e_{1}= & \cos \theta_{1} e_{2}+P_{2} T e_{1}, T e_{2}=-\cos \theta_{1} e_{1}+P_{2} T e_{2}, \ldots, \\
T e_{2 m-1}= & \cos \theta_{1} e_{2 m}+P_{2} T e_{2 m-1}, T e_{2 m}=-\cos \theta_{1} e_{2 m-1}+P_{2} T e_{2 m} \\
T e_{2 m+1}= & \cos \theta_{2} e_{2 m+2}+P_{1} T e_{2 m+1}, T e_{2 m+2}=-\cos \theta_{2} e_{2 m+1}+P_{1} T e_{2 m+2} \\
& , \ldots, T e_{2 n}=-\cos \theta_{2 n-1} e_{2 n}+P_{1} T e_{2 n-1}
\end{aligned}
$$

Since both $D_{1}$ and $D_{2}$ are slant distributions, we note that $P_{i} T e_{\ell+2}$ is orthogonal to both $e_{\ell}$ and $e_{\ell+1}$ vectors, where $e_{\ell}, e_{\ell+1}$ and $e_{\ell+2}$ are any mutually orthogonal vectors in $D_{i}, i \in\{1,2\}$.

If $M$ is a semi-slant submanifold of $\widetilde{M}$, then $\theta_{1}=0$. Thus, we have

$$
\left\langle J X_{1}, Y_{2}\right\rangle=0
$$

for all $X_{1} \in \Gamma\left(D_{1}\right)$ and $Y_{2} \in \Gamma\left(D_{2}\right)$. Taking into consideration (3.8) and (3.9), we can choose an orthonormal basis $\left\{e_{1}, \ldots, e_{2 n}\right\}$ of semi-slant submanifolds satisfies that

$$
\begin{aligned}
T e_{1} & =e_{2}, \ldots, T e_{2 m-1}=e_{2 m} \\
T e_{2 m+1} & =\cos \theta_{2} e_{2 m+2}, \ldots, T e_{2 n-1}=\cos \theta_{2} e_{2 n} .
\end{aligned}
$$


If $M$ is a hemi-slant submanifold of $\widetilde{M}$, then $\theta_{2}=\frac{\pi}{2}$. Thus, we have

$$
\left\langle J X_{2}, Y_{2}\right\rangle=0
$$

for all $X_{2}, Y_{2} \in \Gamma\left(D_{2}\right)$. From (3.8) and (3.11), we can choose an orthonormal basis $\left\{e_{1}, \ldots, e_{2 n}\right\}$ of hemi-slant submanifolds satisfying that

$$
\begin{aligned}
\left\|P_{1} T e_{j}\right\|^{2} & =\left\|T e_{j}\right\|^{2}, \quad i \in\{1, \ldots, 2 m\}, \\
\left\|P_{2} T e_{i}\right\|^{2} & =0, \quad j \in\{2 m+1, \ldots, 2 n\} .
\end{aligned}
$$

Now, we shall need the following lemma:

Lemma 3.4 ([5]). Let $M$ be a $\left(\theta_{1}, \theta_{2}\right)$ bi-slant submanifold of an almost Hermitian manifold $\widetilde{M}$. Given $\theta \in\left[0, \frac{\pi}{2}\right], M$ is $\theta$-slant if and only if the following equations hold:

$$
\begin{array}{r}
P_{2} T P_{1} T P_{1}+P_{2} T P_{2} T P_{1}=0, \\
P_{1} T P_{1} T P_{2}+P_{1} T P_{2} T P_{2}=0, \\
P_{1} T P_{2} T P_{1}=\left(\cos ^{2} \theta_{1}-\cos ^{2} \theta\right) P_{1}, \\
P_{2} T P_{1} T P_{2}=\left(\cos ^{2} \theta_{2}-\cos ^{2} \theta\right) P_{2} .
\end{array}
$$

Let $M$ be a $\left(\theta_{1}, \theta_{2}\right)$ bi-slant submanifold. Also, if $M$ is a $\theta$-slant submanifold, then taking into consideration (3.15), we have

$$
\begin{aligned}
\left\|P_{2} T e_{i}\right\|^{2}=\left\langle P_{2} T e_{i}, P_{2} T e_{i}\right\rangle & =-\left\langle e_{i}, T P_{2} T e_{i}\right\rangle \\
& =-\left\langle e_{i}, P_{1} T P_{2} T e_{i}\right\rangle \\
& =\cos ^{2} \theta-\cos ^{2} \theta_{1}
\end{aligned}
$$

for $i \in\{1, \ldots, 2 m\}$. Using the similar way, we have

$$
\left\|P_{1} T e_{j}\right\|^{2}=\cos ^{2} \theta-\cos ^{2} \theta_{2}
$$

for $j \in\{2 m+1, \ldots, 2 n\}$. From (3.8), (3.17) and (3.18), if $M$ is a slant submanifold with $\theta_{1}=\theta_{2}=$ $\theta$, then we can choose orthonormal basis $\left\{e_{1}, \ldots, e_{2 n}\right\}$ of $T_{p} M$ such that

$$
T e_{1}=\cos \theta e_{2}, \ldots, T e_{2 n-1}=\cos \theta e_{2 n} .
$$

We note that the orthonormal basis given in (3.19) was firstly given by B.-Y. Chen in [7].

\section{Ricci curvature}

In this section, we are going to give an inequality involving Ricci curvature of bi-slant submanifold on generalized complex space form and study this inequality for semi-slant submanifold, hemi-slant submanifold and slant submanifold of generalized complex space forms. 
Theorem 4.1. Let $M$ be a $2 n$-dimensional $\left(\theta_{1}, \theta_{2}\right)$ bi-slant submanifold of a $2 k$-dimensional generalized complex space form. Then

(a) For $X \in T_{p}^{1} M=\left\{X \in T_{p} M \mid\langle X, X\rangle=1\right\}$, it follows that

$$
\begin{aligned}
\operatorname{Ric}(X) \leq & n^{2}\|H\|^{2}+\frac{c+3 \alpha}{4}(2 n-1)+\frac{3(c-\alpha)}{8}\left(\cos ^{2} \theta_{1}+\cos ^{2} \theta_{2}\right) \\
& \left.+\left\|P_{1} T X_{D_{2}}\right\|^{2}+\left\|P_{2} T X_{D_{1}}\right\|^{2}\right),
\end{aligned}
$$

where $\theta_{1}$ is slant angle of $D_{1}, \theta_{2}$ is slant angle of $D_{2}$ and $\operatorname{Ric}(X)$ is the Ricci curvature of $M$.

(b) The equality case of (4.1) is satisfied by $X \in T_{p}^{1} M$ if and only if

$$
\left\{\begin{array}{l}
\sigma(X, Y)=0, \quad \text { for all } Y \in T_{p} M \text { orthogonal to } X \\
\sigma(X, X)=n H(p) .
\end{array}\right.
$$

(c) The equality case of (4.1) holds for all $X \in T_{p}^{1} M$ if and only if $p$ is a totally geodesic point.

Proof. From (2.4) and (2.5), we have

$$
\begin{aligned}
n^{2}\|H\|^{2}= & \tau(p)-\widetilde{\tau}\left(T_{p} M\right)+\frac{1}{4} \sum_{r=n+1}^{2 k}\left(\sigma_{11}^{r}-\sigma_{22}^{r}-\cdots-\sigma_{n n}^{r}\right)^{2} \\
& +\sum_{r=2 n+1}^{2 k} \sum_{j=2}^{2 n}\left(\sigma_{1 j}^{r}\right)^{2}-\sum_{r=2 n+1}^{2 k} \sum_{1 \neq i<j \leq 2 n}\left(\sigma_{i i}^{r} \sigma_{j j}^{r}-\left(\sigma_{i j}^{r}\right)^{2}\right) .
\end{aligned}
$$

Using

$$
\sum_{r=2 n+1}^{2 k} \sum_{1 \neq i<j \leq 2 n} \sigma_{i i}^{r} \sigma_{j j}^{r}-\left(\sigma_{i j}^{r}\right)^{2}=\sum_{1 \neq i<j \leq 2 n} K_{i j}-\widetilde{K}_{i j}
$$

and

$$
\sum_{1 \neq i<j \leq 2 n} K_{i j}=\tau(p)-\operatorname{Ric}\left(e_{1}\right)
$$

we obtain

$$
\begin{aligned}
\operatorname{Ric}\left(e_{1}\right)= & n^{2}\|H\|^{2}+\widetilde{\operatorname{Ric}}_{T_{p} M}\left(e_{1}\right)-\sum_{r=2 n+1}^{2 k} \sum_{j=2}^{2 n}\left(\sigma_{1 j}^{r}\right)^{2} \\
& -\frac{1}{4} \sum_{r=2 n+1}^{2 k}\left(\sigma_{11}^{r}-\sigma_{22}^{r}-\cdots-\sigma_{n n}^{r}\right)^{2} .
\end{aligned}
$$

Since we can choose $e_{1}=X$ as any unit vector in $T_{p}^{1} M$, we get the following inequality:

$$
\operatorname{Ric}(X) \leq n^{2}\|H\|^{2}+\widetilde{\operatorname{Ric}}_{\left(T_{p} M\right)}(X) .
$$

Now, we calculate $\widetilde{\operatorname{Ric}}_{\left(T_{p} M\right)}(X)$. Let $p \in M$ and $\left\{e_{1}, \ldots, e_{2 m}, e_{2 m+1}, \ldots, e_{2 n}\right\}$ be orthonormal basis of $M$ such that $\left\{e_{1}, \ldots, e_{2 m}\right\}$ is an orthonormal basis of $D_{1}$ and $\left\{e_{2 m+1}, \ldots, e_{2 n}\right\}$ is an orthonormal basis of $D_{2}$. From (2.9) and (3.8), we have the following equalities:

$$
\widetilde{\operatorname{Ric}}_{D_{1}}\left(e_{1}\right)=\sum_{j=1}^{2 m} \widetilde{g}\left(\widetilde{R}\left(e_{1}, e_{j}\right) e_{j}, e_{1}\right)
$$




$$
\begin{aligned}
& =\frac{c+3 \alpha}{4}(2 m-1)+\frac{3(c-\alpha)}{4} \cos ^{2} \theta_{1}, \\
& \widetilde{R i}_{D_{2}}\left(e_{2 m+1}\right)=\sum_{j=2 m+2}^{2 n} \widetilde{g}\left(\widetilde{R}\left(e_{2 m+1}, e_{j}\right) e_{j}, e_{2 m+1}\right) \\
& =\frac{c+3 \alpha}{4}(2 n-2 m-1)+\frac{3(c-\alpha)}{4} \cos ^{2} \theta_{2}, \\
& \sum_{j=2 m+1}^{2 n} \widetilde{g}\left(\widetilde{R}\left(e_{1}, e_{j}\right) e_{j}, e_{1}\right)=\frac{c+3 \alpha}{4}(2 n-2 m)+\frac{3(c-\alpha)}{4} \sum_{j=2 m+1}^{2 n}\left\langle P_{2} T e_{1}, e_{j}\right\rangle^{2}, \\
& \sum_{j=1}^{2 m} \widetilde{g}\left(\widetilde{R}\left(e_{2 m+1}, e_{j}\right) e_{j}, e_{2 m+1}\right)=\frac{c+3 \alpha}{4}(2 m)+\frac{3(c-\alpha)}{4} \sum_{j=1}^{2 m}\left\langle P_{1} T e_{2 m+1}, e_{j}\right\rangle^{2}, \\
& \sum_{j=1}^{2 m} \widetilde{g}\left(\widetilde{R}\left(e_{1}, e_{j}\right) e_{j}, e_{2 m+1}\right)=\frac{3(c-\alpha)}{4} \sum_{j=1}^{2 m}\left\langle T e_{1}, e_{j}\right\rangle\left\langle T e_{2 m+1}, e_{j}\right\rangle \\
& =\frac{3(c-\alpha)}{4}\left\langle J e_{1}, J e_{2 m+1}\right\rangle \\
& =0 \text {. }
\end{aligned}
$$

Now, we choose $X=\frac{1}{\sqrt{2}}\left(e_{1}+e_{2 m+1}\right)$. Then $\|X\|=1$ and $X \in T_{p}^{1} M$. Thus, we get

$$
\begin{aligned}
\widetilde{R i c}_{T_{p} M}(X)= & \frac{1}{2}\left\{{\widetilde{R i c_{D_{1}}}}\left(e_{1}\right)+\widetilde{\operatorname{Ric}}_{D_{2}}\left(e_{2 m+1}\right)\right. \\
& \left.+\sum_{j=2 m+1}^{2 n} \widetilde{g}\left(\widetilde{R}\left(e_{1}, e_{j}\right) e_{j}, e_{1}\right)+\sum_{j=1}^{2 m} \widetilde{g}\left(\widetilde{R}\left(e_{2 m+1}, e_{j}\right) e_{j}, e_{2 m+1}\right)\right\} \\
& +\sum_{j=1}^{2 m} \widetilde{g}\left(\widetilde{R}\left(e_{1}, e_{j}\right) e_{j}, e_{2 m+1}\right) .
\end{aligned}
$$

If we put (4.8), (4.9), (4.10), (4.11) and (4.12) in (4.13), we have

$$
\begin{aligned}
\widetilde{\text { Ric }}_{T_{p} M}(X)= & \frac{c+3 \alpha}{4}(2 n-1)+\frac{3(c-\alpha)}{8}\left(\cos ^{2} \theta_{1}+\cos ^{2} \theta_{2}\right) \\
& \left.+\sum_{j=2 m+1}^{2 n}\left\langle P_{2} T e_{1}, e_{j}\right\rangle^{2}+\sum_{j=1}^{2 m}\left\langle P_{1} T e_{2 m+1}, e_{j}\right\rangle^{2}\right\} .
\end{aligned}
$$

From (4.14) and (4.7), we get (4.1). The equality in (4.1) is valid if and only if

$$
\sigma_{12}^{r}=\cdots=\sigma_{12 n}^{r}=0 \text { and } \sigma_{11}^{r}=\sigma_{22}^{r}+\cdots+\sigma_{2 n 2 n}^{r}, \quad r \in\{2 n+1, \ldots, 2 k\},
$$

which is equivalent to (4.2).

Now, we prove the statement (c). Assuming the equality case of (4.1) for all $X \in T_{p}^{1} M$, in view of (4.15), for each $r \in\{2 n+1, \ldots, 2 k\}$, we have

$$
\sigma_{i j}^{r}=0, \quad i \neq j
$$




$$
2 \sigma_{i i}^{r}=\sigma_{11}^{r}+\sigma_{22}^{r}+\cdots+\sigma_{2 n 2 n}^{r}, \quad i \in\{1, \ldots, 2 n\} .
$$

From (4.17), we have $2 \sigma_{11}^{r}=2 \sigma_{22}^{r}=\cdots=2 \sigma_{2 n 2 n}^{r}=\sigma_{11}^{r}+\sigma_{22}^{r}+\cdots+\sigma_{2 n 2 n}^{r}$, which implies that

$$
2(n-1)\left(\sigma_{11}^{r}+\sigma_{22}^{r}+\cdots+\sigma_{2 n 2 n}^{r}\right)=0 .
$$

Since $n \neq 1, \sigma_{11}^{r}+\sigma_{22}^{r}+\cdots+\sigma_{n n}^{r}=0$ is valid. Then in view of (4.17), we get $\sigma_{i i}^{r}=0$ for all $i \in$ $\{1, \ldots, n\}$. This together with (4.16) gives $\sigma_{i j}^{r}=0$ for all $i, j \in\{1, \ldots, 2 n\}$ and $r \in\{2 n+1, \ldots, 2 k\}$, that is, $p$ is a totally geodesic point. The proof of the converse part is straightforward.

From Theorem 4.1, we get the following corollaries:

\section{Corollary 4.2.}

(a) Let $M$ be a $2 n$-dimensional submanifold of a generalized complex space form $\widetilde{M}(c, \alpha)$. We

\begin{tabular}{|c|c|c|c|}
\hline & $\widetilde{M}$ & $M$ & Inequality \\
\hline (1) & $\widetilde{M}(c, \alpha)$ & bi - slant & $\begin{aligned} \operatorname{Ric}(X) \leq & n^{2}\|H\|^{2}+\frac{c+3 \alpha}{4}(2 n-1)+\frac{3(c-\alpha)}{8}\left(\cos ^{2} \theta_{1}+\cos ^{2} \theta_{2}\right. \\
& \left.+\left\|P_{1} T X_{D_{2}}\right\|^{2}+\left\|P_{2} T X_{D_{1}}\right\|^{2}\right)\end{aligned}$ \\
\hline (2) & $\widetilde{M}(c, \alpha)$ & semi-slant & $\operatorname{Ric}(X) \leq n^{2}\|H\|^{2}+\frac{c+3 \alpha}{4}(2 n-1)+\frac{3(c-\alpha)}{8}\left(1+\cos ^{2} \theta_{2}\right)$ \\
\hline (3) & $\widetilde{M}(c, \alpha)$ & hemi-slant & $\begin{aligned} \operatorname{Ric}(X) \leq & n^{2}\|H\|^{2}+\frac{c+3 \alpha}{4}(2 n-1)+\frac{3(c-\alpha)}{8}\left(\cos ^{2} \theta_{1}+\left\|T X_{D_{2}}\right\|^{2}\right. \\
& \left.+\left\|P_{2} T X_{D_{1}}\right\|^{2}\right)\end{aligned}$ \\
\hline (4) & $\widetilde{M}(c, \alpha)$ & CR & $\operatorname{Ric}(X) \leq n^{2}\|H\|^{2}+\frac{c+3 \alpha}{4}(2 n-1)+\frac{3(c-\alpha)}{8}$ \\
\hline (5) & $\widetilde{M}(c, \alpha)$ & $\begin{array}{l}\boldsymbol{\theta} \text { - slant with } \\
\boldsymbol{\theta}_{1}=\boldsymbol{\theta}_{2}=\boldsymbol{\theta} \text { or } \\
\boldsymbol{\theta}_{1}=\boldsymbol{\theta}_{2} \neq \boldsymbol{\theta}\end{array}$ & $\operatorname{Ric}(X) \leq n^{2}\|H\|^{2}+\frac{c+3 \alpha}{4}(2 n-1)+\frac{3(c-\alpha)}{4} \cos ^{2} \theta$ \\
\hline (6) & $\widetilde{M}(c, \alpha)$ & invariant & $\operatorname{Ric}(X) \leq n^{2}\|H\|^{2}+\frac{c+3 \alpha}{4}(2 n-1)+\frac{3(c-\alpha)}{4}$ \\
\hline (7) & $\widetilde{M}(c, \alpha)$ & anti-invariant & $\operatorname{Ric}(X) \leq n^{2}\|H\|^{2}+\frac{c+3 \alpha}{4}(2 n-1)$ \\
\hline
\end{tabular}
have the following table: 
(b) The equality case of inequalities in the previous table is satisfied for $X \in T_{p}^{1} M$ if and only if

$$
\left\{\begin{array}{l}
\sigma(X, Y)=0, \quad \text { for all } Y \in T_{p} M \text { orthogonal to } X \\
2 \sigma(X, X)=n H(p) .
\end{array}\right.
$$

If $H(p)=0$, then $X \in T_{p}^{1} M$ satisfies the equality case of inequalities in the previous table if and only if $X \in \mathscr{N}_{p}=\left\{X \in T_{p} M: \sigma(X, Y)=0, \forall Y \in T_{p} M\right\}$.

(c) The equality case of inequalities (1)-(4) is satisfied for all $X \in T_{p}^{1} M$ if and only if $p$ is a totally geodesic point.

(d) The equality case of inequalities (5)-(7) is satisfied for all $X \in T_{p}^{1} M$ if and only if either $p$ is a totally geodesic point or $n=1$ and $p$ is a totally umbilical point.

Proof. From Theorem 4.1, we have the inequality (1) immediately. Taking (3.10) into consideration, we have

$$
P_{1} T X_{D_{2}}=P_{2} T X_{D_{1}}=0
$$

where $X=X_{D_{1}}+X_{D_{2}}$ for $X_{D_{1}} \in D_{1}$ and $X_{D_{2}} \in D_{2}$. If we write (4.18) and (3.12) in the inequality (1), we get the inequalities (2) and (3), respectively. Next, putting $\theta_{2}=\frac{\pi}{2}$ in the inequality (2), we have the inequality (4) or putting $\theta_{1}=0$ in the inequality (3), then

$$
T X_{D_{2}}=P_{2} T X_{D_{1}}=0 \text {. }
$$

Thus, we have the inequality (4) again. If we put (3.17) and (3.18) in the inequality (1), we get the inequality (5). Writing $\theta=0$ and $\theta=\frac{\pi}{2}$ in the inequality (5), we obtain the inequalities (6) and (7), respectively. Hence proof of the (a) is complete. The statements (b-d) are straightforward from Theorem 4.1 .

Remark 4.3. The inequalities (5-7) on the above table were proved by S. Hong and M. M. Tripathi in [11]. The authors also studied generic submanifolds of generalized complex space forms. Since generic submanifolds don't contain slant distributions, we note that bi-slant submanifolds are not a particular case of generic submanifold.

\section{Corollary 4.4.}

(a) Let $M$ be a $2 n$-dimensional submanifold of a complex space form $\widetilde{M}(4 c)$. We have the following table: 


\begin{tabular}{|c|c|c|c|}
\hline & $\widetilde{M}$ & $M$ & Inequality \\
\hline (1) & $\widetilde{M}(4 c)$ & bi-slant & $\begin{aligned} \operatorname{Ric}(X) \leq & n^{2}\|H\|^{2}+(2 n-1) c+\frac{3 c}{2}\left(\cos ^{2} \theta_{1}+\cos ^{2} \theta_{2}\right. \\
& \left.+\left\|P_{1} T X_{D_{2}}\right\|^{2}+\left\|P_{2} T X_{D_{1}}\right\|^{2}\right)\end{aligned}$ \\
\hline (2) & $\widetilde{M}(4 c)$ & semi - slant & $\operatorname{Ric}(X) \leq n^{2}\|H\|^{2}+(2 n-1) c+\frac{3 c}{2}\left(1+\cos ^{2} \theta_{2}\right)$ \\
\hline (3) & $\widetilde{M}(4 c)$ & hemi - slant & $\begin{aligned} \operatorname{Ric}(X) \leq & n^{2}\|H\|^{2}+(2 n-1) c+\frac{3 c}{2}\left(\cos ^{2} \theta_{1}+\left\|T X_{D_{2}}\right\|^{2}\right. \\
& \left.+\left\|P_{2} T X_{D_{1}}\right\|^{2}\right)\end{aligned}$ \\
\hline (4) & $\widetilde{M}(4 c)$ & CR & $\operatorname{Ric}(X) \leq n^{2}\|H\|^{2}+2(n+1) c+\frac{3 c}{2}$ \\
\hline (5) & $\widetilde{M}(4 c)$ & $\begin{array}{l}\boldsymbol{\theta} \text { - slant with } \\
\boldsymbol{\theta}_{1}=\boldsymbol{\theta}_{2}=\boldsymbol{\theta} \text { or } \\
\boldsymbol{\theta}_{1}=\boldsymbol{\theta}_{2} \neq \boldsymbol{\theta}\end{array}$ & $\operatorname{Ric}(X) \leq n^{2}\|H\|^{2}+(2 n-1) c+3 c \cos ^{2} \theta$ \\
\hline (6) & $\widetilde{M}(4 c)$ & anti-invariant & $\operatorname{Ric}(X) \leq n^{2}\|H\|^{2}+(2 n-1) c$ \\
\hline
\end{tabular}

(b) The equality case of inequalities in the previous table is satisfied for $X \in T_{p}^{1} M$ if and only if

$$
\left\{\begin{array}{l}
\sigma(X, Y)=0, \quad \text { for all } Y \in T_{p} M \text { orthogonal to } X, \\
2 \sigma(X, X)=n H(p) .
\end{array}\right.
$$

If $H(p)=0$, then $X \in T_{p}^{1} M$ satisfies the equality case of inequalities in the previous table if and only if $X \in \mathscr{N}_{p}=\left\{X \in T_{p} M: \sigma(X, Y)=0, \forall Y \in T_{p} M\right\}$.

(c) The equality case of inequalities (1)-(4) is satisfied for all $X \in T_{p}^{1} M$ if and only if $p$ is a totally geodesic point.

(d) The equality case of inequalities (5)-(6) is satisfied for all $X \in T_{p}^{1} M$ if and only if either $p$ is a totally geodesic point or $n=2$ and $p$ is a totally umbilical point.

Remark 4.5. In [13], K. Matsumoto, I. Mihai and A. Oiaga compute the inequalities (5-6). In addition to this, using the fact that every invariant submanifold of a (nearly) Kaehler manifold is minimal, they gave the following corollary.

Corollary 4.6. Let $M$ be an $2 n$-dimensional invariant submanifold of a complex space form $\widetilde{M}(4 c)$. Then 
(a) Any $X \in T_{p}^{1} M=\left\{X \in T_{p} M \mid\langle X, X\rangle=1\right\}$ satisfies

$$
\operatorname{Ric}(X) \leq 2(n+1) c
$$

(b) An $X \in T_{p}^{1} M$ satisfies the equality case of (4.20) if and only if $X \in \mathscr{N}_{p}$.

(c) The equality case of (4.20) is satisfied for all $X \in T_{p}^{1} M$ if and only if $p$ is a totally geodesic point.

\section{Scalar curvature and $\delta$-invariant}

Now, we recall the following definition of B.-Y. Chen and the following Lemma for future uses [8].

Definition 5.1. Let $M$ be a submanifold of a Riemannian manifold $\widetilde{M}$. The Chen invariant at a point $p \in M$, denoted by $\delta_{M}(p)$, is defined by

$$
\delta_{M}(p)=\tau(p)-\inf (K)(p),
$$

where $\tau(p)$ is the scalar curvature and

$$
\inf (K)(p)=\inf \left\{K(\Pi): K(\Pi) \text { is a plane section of } T_{p} M\right\}
$$

Lemma 5.2. If $n>k \geq 2$ and $a_{1}, \ldots, a_{n}$, a are real numbers such that

$$
\left(\sum_{i=1}^{n} a_{i}\right)^{2}=(n-1)\left(\sum_{i=1}^{n} a_{i}^{2}+a\right),
$$

then

$$
2 a_{1} a_{2} \geq a
$$

with equality holding if and only if

$$
a_{1}+a_{2}=a_{3}=\cdots=a_{n}
$$

Let $\Pi=\operatorname{Span}\{X, Y\}$ be any 2-dimensional plane section of $T_{p} M$. We set

$$
T(\Pi)=\langle T X, Y\rangle^{2} .
$$

Now, we are going to give an optimal inequality involving the Chen invariant as follows: 
Theorem 5.3. Let $M$ be a $2 n$-dimensional $\left(\theta_{1}, \theta_{2}\right)$ bi-slant submanifold of a $2 k$-dimensional generalized complex space form. For any 2-dimensional plane section $\Pi=\operatorname{Span}\{X, Y\}$ in $T_{p} M$, we have

$$
\begin{aligned}
\delta_{M}(p) \leq & \frac{n^{2}(n-2)}{2(n-1)}\|H\|^{2}+\frac{c+3 \alpha}{4}(2 n+1)(n-1)+\frac{3(c-\alpha)}{8}\left[n \cos ^{2} \theta_{1}\right. \\
& \left.+n \cos ^{2} \theta_{2}+\frac{1}{2}\left\|P_{1} T\right\|^{2}+\frac{1}{2}\left\|P_{2} T\right\|^{2}-2 T(\Pi)\right] .
\end{aligned}
$$

The equality case of the inequality (5.4) holds at a point $p \in M$ if and only if there exists an orthonormal basis $\left\{e_{1}, \ldots, e_{2 n}\right\}$ of $T_{p} M$ and an orthonormal basis $\left\{e_{2 n+1}, \ldots, e_{2 k}\right\}$ of $T_{p}^{\perp} M$ such that the shape operators of $M$ have the following forms:

$$
\begin{aligned}
A_{2 n+1} & =\left(\begin{array}{ccccc}
a & 0 & 0 & \ldots & 0 \\
0 & b & 0 & \ldots & 0 \\
0 & 0 & \mu & \ldots & 0 \\
\vdots & \vdots & \vdots & \ddots & 0 \\
0 & 0 & 0 & \ldots & 0
\end{array}\right), a+b=\mu, \\
A_{r} & =\left(\begin{array}{ccccc}
c & d & 0 & \ldots & 0 \\
d & -c & 0 & \ldots & 0 \\
0 & 0 & 0 & \ldots & 0 \\
\vdots & \vdots & \vdots & \ddots & 0 \\
0 & 0 & 0 & \ldots & 0
\end{array}\right), r \in\{2 n+2, \ldots, 2 k\} .
\end{aligned}
$$

Proof. From the Gauss equation and (4.14), we have

$$
\begin{aligned}
2 \tau(p)= & n^{2}\|H\|^{2}-\|\sigma\|^{2}+\frac{c+3 \alpha}{2}(2 n-1) n+\frac{3(c-\alpha)}{8}\left[2 n \cos ^{2} \theta_{1}\right. \\
& \left.+2 n \cos ^{2} \theta_{2}+\left\|P_{1} T\right\|^{2}+\left\|P_{2} T\right\|^{2}\right] .
\end{aligned}
$$

In equation (5.7), if we put

$$
\begin{aligned}
w= & 2 \tau(p)-\frac{n^{2}(n-1)}{n-2}\|H\|^{2}-\frac{c+3 \alpha}{2}(2 n-1) n \\
& -\frac{3(c-\alpha)}{8}\left[2 n \cos ^{2} \theta_{1}+2 n \cos ^{2} \theta_{2}+\left\|P_{1} T\right\|^{2}+\left\|P_{2} T\right\|^{2}\right],
\end{aligned}
$$

we get

$$
n^{2}\|H\|^{2}=(n-1)\left(w+\|\sigma\|^{2}\right) .
$$

If we choose $e_{2 n+1}$ in the direction of the mean curvature vector $H(p)$, then (5.9) gives

$$
\left(\sum_{i=1}^{2 n} \sigma_{i i}^{2 n+1}\right)^{2}=(n-1)\left[\sum_{i=1}^{2 n}\left(\sigma_{i i}^{2 n+1}\right)^{2}+\sum_{i \neq j}\left(\sigma_{i j}^{2 n+1}\right)^{2}+\sum_{r=2 n+2}^{2 k} \sum_{i, j=1}^{2 n}\left(\sigma_{i j}^{r}\right)^{2}+w\right] .
$$


Applying Lemma 5.2 and equation (5.10), we obtain

$$
2 \sigma_{11}^{2 n+1} \sigma_{22}^{2 n+1} \geq \sum_{i \neq j}\left(\sigma_{i j}^{2 n+1}\right)^{2}+\sum_{r=2 n+2}^{2 k} \sum_{i, j=1}^{2 n}\left(\sigma_{i j}^{r}\right)^{2}+w .
$$

Therefore, we have

$$
\begin{aligned}
K(\Pi)= & \frac{c+3 \alpha}{4}+\frac{3(c-\alpha)}{4} T(\Pi)+\sum_{r=2 n+1}^{2 k}\left[\sigma_{11}^{r} \sigma_{22}^{r}-\left(\sigma_{12}\right)^{2}\right] \\
\geq & \frac{c+3 \alpha}{4}+\frac{3(c-\alpha)}{4} T(\Pi)+\frac{1}{2}\left[\sum_{i \neq j}\left(\sigma_{i j}^{2 n+1}\right)^{2}\right. \\
& \left.+\sum_{r=2 n+2}^{2 k} \sum_{i, j=1}^{2 n}\left(\sigma_{i j}^{r}\right)^{2}+w\right]+\sum_{r=2 n+2}^{2 k} \sigma_{11}^{r} \sigma_{22}^{r}-\sum_{r=2 n+2}^{2 k}\left(\sigma_{12}\right)^{2} \\
= & \frac{c+3 \alpha}{4}+\frac{3(c-\alpha)}{4} T(\Pi)+\frac{1}{2} \sum_{i \neq j}\left(\sigma_{i j}^{2 n+1}\right)^{2} \\
& +\frac{1}{2} \sum_{r=2 n+2}^{2 k} \sum_{i, j=1}^{2 n}\left(\sigma_{i j}^{r}\right)^{2}+\frac{1}{2} \sum_{r=2 n+2}^{2 k}\left(\sigma_{11}^{r}+\sigma_{22}^{r}\right)^{2} \\
& +\sum_{j>2}\left[\left(\sigma_{1 j}^{2 n+1}\right)^{2}+\left(\sigma_{2 j}^{2 n+1}\right)^{2}\right]+\frac{w}{2} \\
\geq & \frac{c+3 \alpha}{4}+\frac{3(c-\alpha)}{4} T(\Pi)+\frac{w}{2} .
\end{aligned}
$$

From (5.8) and (5.12), we have

$$
\begin{aligned}
\inf K(\Pi) \geq & \frac{c+3 \alpha}{4}+\frac{3(c-\alpha)}{4} T(\Pi)+\tau(p)-\frac{n^{2}(n-1)}{n-2}\|H\|^{2} \\
& -\frac{3(c-\alpha)}{8}\left(2 n \cos ^{2} \theta_{1}+2 n \cos ^{2} \theta_{2}+\left\|P_{1} T\right\|^{2}\right. \\
& \left.+\left\|P_{2} T\right\|^{2}\right) .
\end{aligned}
$$

From (5.1) and (5.13), we get (5.4).

The equality case of (5.4) is satisfied at $p \in M$ if and only if

$$
\begin{aligned}
& \sigma_{i j}^{2 n+1}=0, \forall i \neq j, i, j>2, \\
& \sigma_{i j}^{r}=0, \forall i \neq j, i, j>2, r \in\{2 n+1, \ldots, 2 k\}, \\
& \sigma_{11}^{r}+\sigma_{22}^{r}=0, \forall r \in\{2 n+2, \ldots, 2 k\}, \\
& \sigma_{1 j}^{2 n+1}=\sigma_{2 j}^{2 n+1}=0, \forall j>2, \\
& \sigma_{11}^{2 n+1}=\sigma_{22}^{2 n+1}=\sigma_{33}^{2 n+1}=\ldots=\sigma_{2 n 2 n}^{2 n+1},
\end{aligned}
$$

which shows that the shape operators of $M$ at $p \in M$ take the form of (5.5) and (5.6).

From Theorem 5.3, we get the following corollaries: 
Corollary 5.4. Let $M$ be a submanifold of a generalized complex space form $\widetilde{M}(c, \alpha)$. For any 2-dimensional plane section $\Pi$ in $T_{p} M$, we have the following table:

\begin{tabular}{|c|c|c|c|}
\hline & $\widetilde{M}$ & $M$ & Inequality \\
\hline (1) & $\widetilde{M}(c, \alpha)$ & bi-slant & $\begin{aligned} \delta_{M}(p) \leq & \frac{n^{2}(n-2)}{2(n-1)}\|H\|^{2}+\frac{c+3 \alpha}{4}(2 n+1)(n-1)+\frac{3(c-\alpha)}{8}\left[n \cos ^{2} \theta_{1}\right. \\
& \left.+n \cos ^{2} \theta_{2}+\frac{1}{2}\left\|P_{1} T\right\|^{2}+\frac{1}{2}\left\|P_{2} T\right\|^{2}-2 T(\Pi)\right]\end{aligned}$ \\
\hline (2) & $\widetilde{M}(c, \alpha)$ & semi - slant & $\begin{aligned} \delta_{M}(p) \leq & \frac{n^{2}(n-2)}{2(n-1)}\|H\|^{2}+\frac{c+3 \alpha}{4}(2 n+1)(n-1)+\frac{3(c-\alpha)}{8}\left[n\left(1+\cos ^{2} \theta_{2}\right)\right. \\
& -2 T(\Pi)]\end{aligned}$ \\
\hline (3) & $\widetilde{M}(c, \alpha)$ & hemi-slant & $\begin{aligned} \delta_{M}(p) \leq & \frac{n^{2}(n-2)}{2(n-1)}\|H\|^{2}+\frac{c+3 \alpha}{4}(2 n+1)(n-1)+\frac{3(c-\alpha)}{8}\left[n \cos ^{2} \theta_{1}+\frac{1}{2}\|T\|^{2}\right. \\
& \left.+\frac{1}{2}\left\|P_{2} T\right\|^{2}-2 T(\Pi)\right]\end{aligned}$ \\
\hline (4) & $\widetilde{M}(c, \alpha)$ & CR & $\delta_{M}(p) \leq \frac{n^{2}(n-2)}{2(n-1)}\|H\|^{2}+\frac{c+3 \alpha}{4}(2 n+1)(n-1)+\frac{3(c-\alpha)}{8}[n-2 T(\Pi)]$ \\
\hline (5) & $\widetilde{M}(c, \alpha)$ & $\begin{array}{l}\boldsymbol{\theta}-\text { slant with } \\
\boldsymbol{\theta}_{1}=\boldsymbol{\theta}_{2}=\boldsymbol{\theta} \text { or } \\
\boldsymbol{\theta}_{1}=\boldsymbol{\theta}_{2} \neq \boldsymbol{\theta}\end{array}$ & $\delta_{M}(p) \leq \frac{n^{2}(n-2)}{2(n-1)}\|H\|^{2}+\frac{c+3 \alpha}{4}(2 n+1)(n-1)+\frac{3(c-\alpha)}{4}\left[n \cos ^{2} \theta-T(\Pi)\right]$ \\
\hline (6) & $\widetilde{M}(c, \alpha)$ & invariant & $\delta_{M}(p) \leq \frac{n^{2}(n-2)}{2(n-1)}\|H\|^{2}+\frac{c+3 \alpha}{4}(2 n+1)(n-1)+\frac{3(c-\alpha)}{4}[n-T(\Pi)]$ \\
\hline (7) & $\widetilde{M}(c, \alpha)$ & anti-invariant & $\delta_{M}(p) \leq \frac{n^{2}(n-2)}{2(n-1)}\|H\|^{2}+\frac{c+3 \alpha}{4}(2 n+1)(n-1)$ \\
\hline
\end{tabular}

The equality case of the inequalities holds at a point $p \in M$ if and only if the shape operators of $M$ take the form of (5.5) and (5.6).

Remark 5.5. The inequalities (5-7) were proved by J-S. Kim, Y-M. Song and M. M. Tripathi in [12] and by A. Mihai in [14].

Corollary 5.6. Let $M$ be a submanifold of a complex space form $\widetilde{M}(4 c)$. For any 2-dimensional 
plane section $\Pi$ in $T_{p} M$, we have the following table:

\begin{tabular}{|c|c|c|c|}
\hline & $\widetilde{M}$ & $M$ & Inequality \\
\hline (1) & $\widetilde{M}(c, \alpha)$ & bi-slant & $\begin{aligned} \delta_{M}(p) \leq & \frac{n^{2}(n-2)}{2(n-1)}\|H\|^{2}+(2 n+1)(n-1) c+\frac{3 c}{2}\left[n \cos ^{2} \theta_{1}\right. \\
& \left.+n \cos ^{2} \theta_{2}+\frac{1}{2}\left\|P_{1} T\right\|^{2}+\frac{1}{2}\left\|P_{2} T\right\|^{2}-2 T(\Pi)\right]\end{aligned}$ \\
\hline (2) & $\widetilde{M}(c, \alpha)$ & semi-slant & $\begin{aligned} \delta_{M}(p) \leq & \frac{n^{2}(n-2)}{2(n-1)}\|H\|^{2}+(2 n+1)(n-1) c+\frac{3 c}{2}\left[n\left(1+\cos ^{2} \theta_{2}\right)\right. \\
& -2 T(\Pi)]\end{aligned}$ \\
\hline (3) & $\widetilde{M}(c, \alpha)$ & hemi-slant & $\begin{aligned} \delta_{M}(p) \leq & \frac{n^{2}(n-2)}{2(n-1)}\|H\|^{2}+(2 n+1)(n-1) c+\frac{3 c}{2}\left[n \cos ^{2} \theta_{1}+\frac{1}{2}\|T\|^{2}\right. \\
& \left.+\frac{1}{2}\left\|P_{2} T\right\|^{2}-2 T(\Pi)\right]\end{aligned}$ \\
\hline$(4)$ & $\widetilde{M}(c, \alpha)$ & CR & $\delta_{M}(p) \leq \frac{n^{2}(n-2)}{2(n-1)}\|H\|^{2}+(2 n+1)(n-1) c+\frac{3 c}{2}[n-2 T(\Pi)]$ \\
\hline (5) & $\widetilde{M}(c, \alpha)$ & $\begin{array}{l}\boldsymbol{\theta}-\text { slant with } \\
\boldsymbol{\theta}_{1}=\boldsymbol{\theta}_{2}=\boldsymbol{\theta} \text { or } \\
\boldsymbol{\theta}_{1}=\boldsymbol{\theta}_{2} \neq \boldsymbol{\theta}\end{array}$ & $\delta_{M}(p) \leq \frac{n^{2}(n-2)}{2(n-1)}\|H\|^{2}+(2 n+1)(n-1) c+3 c\left[n \cos ^{2} \theta-T(\Pi)\right]$ \\
\hline (6) & $\widetilde{M}(c, \alpha)$ & anti-invariant & $\delta_{M}(p) \leq \frac{n^{2}(n-2)}{2(n-1)}\|H\|^{2}+(2 n+1)(n-1) c$ \\
\hline
\end{tabular}

The equality case of the inequalities holds at a point $p \in M$ if and only if the shape operators of $M$ take the form of (5.5) and (5.6).

Corollary 5.7. Let $M$ be a $2 n$-dimensional invariant submanifold of a complex space form $\widetilde{M}(4 c)$. For any 2-dimensional plane section $\Pi$ in $T_{p} M$, we have

$$
\delta_{M}(p) \leq(2 n+1)(n-1) c+3 c[n-T(\Pi)] .
$$

The equality case of (5.15) satisfied at $p \in M$ if and only if the shape operators of $M$ take form as (5.5) and (5.6). 


\section{Acknowledgement}

The second author of this work is supported by 113F388 coded project in the Scientific and Technological Research Council of Turkey (TÜBİTAK). The authors would like to express their warm thanks to the referee for his/her constructive suggestions which improved the content and presentation of the paper.

\section{References}

[1] J. L. Cabrerizo, A. Carriazo, L. M. Fernández and M. Fernández, Slant submanifolds in Sasakian manifolds, Glasg. Math. J., 42 (2000), 125-138.

[2] E. Calabi, Isometric embeddings of complex manifolds, Ann. of Math., 58 (1953), 1-23.

[3] E. Calabi, Metric Riemann surfaces, Ann. of Math. Studies, 30 (1953), 77-85.

[4] A. Carriazo, New developments in slant submanifolds theory, Applicable Mathematics in the Golden Age (Edited by J.C. Misra), Narosa Publishing House (2002), 339-356.

[5] A. Carriazo, Bi-slant immersions, Proceedings ICRAMS (2000), 88-97.

[6] B.-Y. Chen, K. Ogiue, On totally real submanifolds, Trans. Amer. Math. Soc., 193 (1974), 257-266.

[7] B.-Y. Chen, Geometry of slant submanifolds, Katholieke Universiteit Leuven, 1990.

[8] B.-Y. Chen, A Riemannian invariant for submanifolds in space forms and its applications. Geometry and Topology of Submanifolds, VI (Leuven, 1993/Brussels, 1993) (NJ: World Scientific Publishing, River Edge) (1994), pp. 58-81 no. 6, 568-578.

[9] A. Gray, Nearly Kähler manifolds, J. Differ. Geo. 4 (1970), 283-309.

[10] H. H. Hacısalihog̃lu, Lineer Cebir, Hacısalihog̃lu Yayınları, Matematik-Geometri Dizisi, 9. Baskı, 2010.

[11] S. Hong, M. M. Tripathi, On Ricci curvature of submanifolds, Int. J. Pure and Appl. Sci. 2 (2005), $227-246$.

[12] J-S. Kim, Y-M. Y-M. Song, M. M. Tripathi, B.-Y. Chen inequalities for submanifolds in generalized complex space forms, Bull. Korean Math. Soc., 40 (2003), 411-423.

[13] K. Matsumoto, I. Mihai, A. Oiaga, Ricci curvature of submanifolds in complex space forms, Rev. Roumanie Math. Pures Appl. 46 (2001), 775-782.

[14] A. Mihai, B.Y. Chen inequalities for slant submanifolds in generalized complex space forms, Radovi Matematički, 12 (2004), 215-231.

[15] P. K. Pandey, R. S. Gupta, A. Sharfuddin, B. Y. Chen's inequalities for bi-slant submanifolds in Kenmotsu space forms, Demonstrario Mathematica 18 (2010), 887-898.

[16] S. S. Shukla, P. K. Rao, B.Y. Chen inequalities for bi-slant submanifolds in generalized complex space forms, The J. of Nonlinear Sci. and Appl. 3 (2010), 282-293.

[17] F. Tricerri, L. Vanhecke, Curvature tensors on almost Hermitian manifolds, Trans. Am. Math. Soc., 267 (1981), 365-398.

[18] L. Vanhecke, Almost Hermitian manifolds with J-invariant Riemann curvature tensor, Rend. Sem. Mat. Univers. Politec. Torino 34 (1975/76), 487-498.

[19] K. Yano, M. Kon, Structures on manifolds, Series in Pure Mathematics, Volume 3, 1984.

Siirt University, Science \& Arts Faculty, Department of Mathematics, Siirt, Turkey.

E-mail: mehmetgulbahar85@gmail.com

İnönü University, Science \& Arts Faculty, Department of Mathematics, Malatya, Turkey.

E-mail: erol.kilic@inonu.edu.tr

İnönü University, Science \& Arts Faculty, Department of Mathematics, Malatya, Turkey.

E-mail: sadik.keles@inonu.edu.tr 